\title{
Robust control of drag and lateral dynamic response for road vehicles exposed to cross-wind gusts
}

\author{
Jens Pfeiffer ${ }^{1}\left[\right.$ : $\cdot$ Rudibert King $^{1}$
}

Received: 4 September 2017 / Revised: 28 November 2017 / Accepted: 9 December 2017 / Published online: 9 February 2018

(c) The Author(s) 2018. This article is an open access publication

\begin{abstract}
A robust closed-loop active flow control strategy for road vehicles under unsteady cross-wind conditions is presented. It is designed based on black-box models identified from experimental data for a 3D bluff body equipped with Coanda actuators along the rear edges. The controller adjusts the blowing rates of the actuators individually, achieving a drag reduction of about $15 \%$ while simultaneously improving cross-wind sensitivity. Hereby, the lateral vehicle dynamics and driver behavior are taken into account and replicated in the wind tunnel via a novel model support system. The effectiveness of the control strategy is demonstrated via cross-wind gust experiments.
\end{abstract}

\section{Introduction}

Most wind-tunnel investigations of road vehicles are conducted under low-turbulent free-stream conditions, mainly with the objective to reduce aerodynamic drag and thus increase fuel efficiency. However, there has been a growing interest in how unsteady flow phenomena affect vehicle aerodynamics. These effects can be classified into three different groups: time-varying external flow conditions, for example, due to side-wind gusts; unsteady effects created by the lateral or vertical vehicle motion; and self-excited flow characteristics such as wake instabilities. Sims-Williams (2011) gives an overview over the related test methods and results obtained by different researchers. Accordingly, cross-wind gusts with scales of 2-20 vehicle lengths are the most critical, because the observed flow phenomena can differ significantly from quasi-steady conditions. Furthermore, lateral vehicle dynamics and driver behavior are sensitive to disturbances in this frequency range. Wagner (2004) and Krantz (2011) point out the importance of lateral vehicle dynamics and driver behavior when assessing a vehicle's cross-wind sensitivity and they present corresponding models.

Approaches to improve the cross-wind sensitivity are mainly limited to passive means such as shape optimization (Krajnović and Basara 2009) or adding airflow break-away

Rudibert King

rudibert.king@tu-berlin.de

1 TU Berlin, Fachgebiet Mess- und Regelungstechnik, Sekr. ER 2-1, Hardenbergstr. 36a, 10623 Berlin, Germany edges (Krantz 2011). Although active flow control (AFC) methods for bluff bodies are gaining more and more attention, they are still mostly investigated under steady, lowturbulent flow conditions with the objective of minimizing aerodynamic drag. Here, most researchers investigate the Ahmed body with actuation on the rear slant (Brunn et al. 2007; Muminović et al. 2008; Aubrun et al. 2011; Joseph et al. 2012; Park et al. 2013; Gilliéron and Kourta 2013), whereas there are only a few reports about the successful application of AFC to square-back configurations. To this end, Littlewood and Passmore (2012) use steady blowing through a single slot at the upper rear edge of a simplified car model for aerodynamic drag reduction, whereas Englar (2001, 2004) applies pneumatic actuation, exploiting the Coanda effect along all four rear edges of a generic trucktrailer configuration. Englar also suggests asymmetric blowing for generating a yaw moment to counter the effects of cross-wind.

Most of the research on AFC for bluff bodies is carried out with open-loop actuation that is operated at constant amplitudes or frequencies. However, AFC, as opposed to passive means, offers the potential to adapt the actuation to changing flow conditions using feedback. In this paper, we present a closed-loop AFC strategy for road vehicles exposed to cross-wind gusts. The feedback controller is designed based on black-box models identified from input-output measurements. It adjusts the blowing rates at the rear edges of a square-back 3D bluff body individually. The controller reduces aerodynamic drag and controls a weighted sum of side-force and yaw-moment coefficients 
to reduce cross-wind sensitivity. Thus, the lateral vehicle dynamics and driver behavior are taken into account. The resulting vehicle motion is calculated in real time based on appropriate dynamic models and is executed online by a novel experimental setup. The effectiveness of the controller in reducing the drag coefficient while improving lateral stability is demonstrated via cross-wind gust experiments.

\section{Experimental setup}

\subsection{Cross-wind tunnel}

The experiments are conducted in a special cross-wind tunnel, the concept of which is based on a similar tunnel used by Dominy and Ryan (1999) at Durham University. Figure 1 shows a CAD representation of the cross-wind facility at Technische Universität Berlin. A blowing open-jet wind tunnel with a nozzle exit width and height of $0.7 \mathrm{~m} \times 0.5 \mathrm{~m}$ is used to generate an axial velocity of up to $20 \mathrm{~m} / \mathrm{s}$ with a turbulence level of less than $3 \%$ in the empty test section. A raised splitter plate $50 \mathrm{~mm}$ above the test-section floor reduces the wind-tunnel boundary layer. The vehicle model is installed at the intersection of the two wind-tunnel axes, at a distance of 0.64 times the vehicle length between the

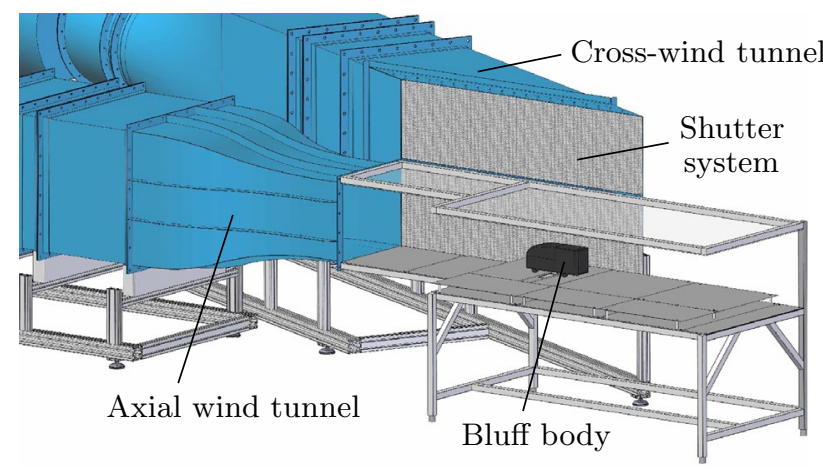

Fig. 1 Cross-wind facility beginning of the raised floor and the front of the body. A second blowing wind tunnel with two outlets, with dimensions of $1.93 \mathrm{~m} \times 0.5 \mathrm{~m}$ each, is installed at an angle of $20^{\circ}$ with respect to the axial jet. When the axial wind-tunnel is set to its maximum velocity of $20 \mathrm{~m} / \mathrm{s}$, cross-wind angles of up to $15^{\circ}$ can be achieved by running the cross-wind fan at maximum speed. During the cross-wind gust experiments, the transient velocity and cross-wind angle are monitored by a Cobra-shaped 5-hole probe (Aeroprobe, PC5-TIP-25-C240-152-025, absolute accuracy better than $0.4^{\circ}$ ) that is installed at the ceiling of the test section above the model front.

For cross-wind gust generation, the outlets of the crosswind tunnel are equipped with 20 shutters that can be turned individually by pneumatic cylinders. Under straight flow conditions, all shutters at the lower outlet are closed, whereas the upper outlet is open to prevent the cross-wind fan from stalling. A microprocessor is used to trigger the shutter blades consecutively with a time delay matching the axial wind-tunnel velocity. This opens the lower outlet starting at the front of the test section and generates a cross-wind gust that travels over the vehicle. Dominy and Ryan (1999) present a detailed study of the gust development in their wind tunnel.

\subsection{Bluff body}

Figure 2 shows the 3D bluff body investigated here. It has been previously studied by the authors (Pfeiffer and King 2012, 2014). The geometry and actuation concept are adopted from Englar (2001, 2004), but the vehicle length was reduced, such that the model represents a small truck or delivery van. Its dimensions are $L=406.5 \mathrm{~mm}$, $H=160 \mathrm{~mm}$, and $W=115 \mathrm{~mm}$, with a wheelbase length of $L_{w b}=230 \mathrm{~mm}$ and a wheel diameter of $44 \mathrm{~mm}$. Drag force $D$, side force $S$, and yaw moment $N$ are defined for the coordinate system located at the center of the wheelbase, as shown in Fig. 2. The 5-hole probe measures dynamic and static pressures $q$ and $p_{s}$, respectively, free-stream velocity $\mathrm{u}_{\infty}$, and cross-wind angle $\beta_{w}$. The
Fig. 2 a Schematic view of the bluff body with $\mathbf{b}$ a cross section depicting the coordinate system, the location of the pressure sensors, and a close-up view of the Coanda actuator geometry
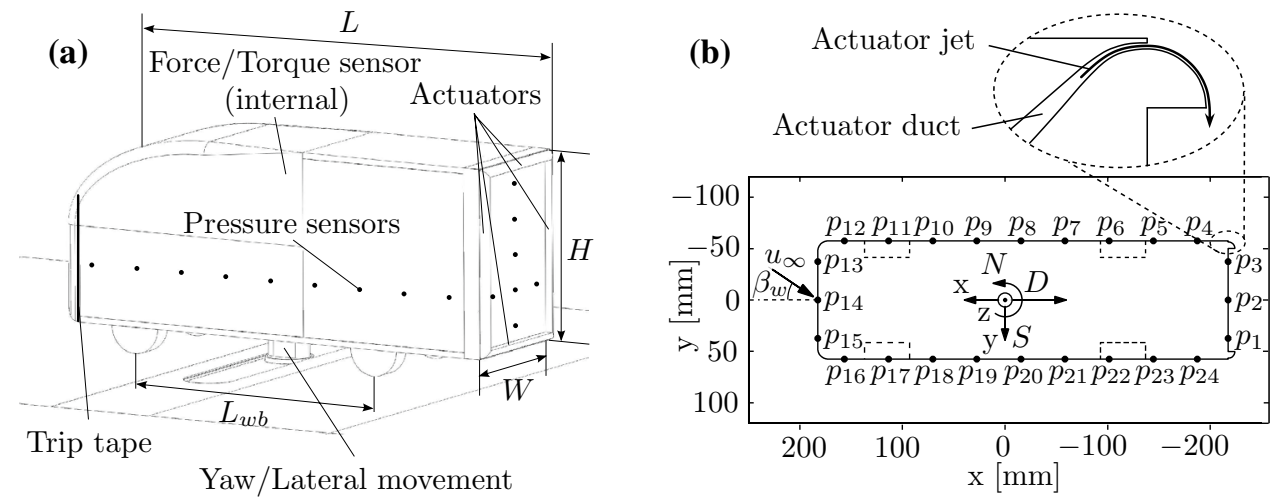
Reynolds number $R e_{L}=\mathrm{u}_{\infty} L / \nu$ is defined for model length $L$ and kinematic viscosity $v$. Trip tapes are installed $15 \mathrm{~mm}$ behind the front of the body. Their efficiency to promote transition location from laminar to turbulent boundary layer was verified by hot-wire measurements. The nondimensional coefficients for the aerodynamic forces and moments are given by the following:

$c_{D}=\frac{D}{\bar{q} A_{B}}, \quad c_{S}=\frac{S}{\bar{q} A_{B}}, \quad c_{N}=\frac{N}{\bar{q} A_{\mathrm{B}} L_{w b}}$,

for the cross-sectional area $A_{\mathrm{B}}$ of the bluff body. Here, we use the time-averaged, nominal dynamic pressure $\bar{q}$ before the cross-wind gust for nondimensionalization. In total, 28 sensors (Sensortechnics, HCLA02X5B, measurement uncertainty due to hysteresis and nonlinearity less than $0.25 \%$ of full scale span) measure the pressure distribution around the vehicle. Of these, 24 are installed along a cross section $72 \mathrm{~mm}$ above the raised floor, whereas seven pressure sensors are located on the bluff body's base, as shown in Fig. 2. The pressure coefficients are calculated by the following:

$c_{p, i}=\frac{p_{i}-\bar{p}_{s}}{\bar{q}}$,

where $p_{i}$ denotes the pressure measured at location $i$, and $\bar{p}_{s}$ refers to the mean, nominal static pressure. Four pneumatic actuators along the vehicle's rear edges are used for active flow control. They run separately and their velocities can be adjusted individually to apply an asymmetric actuation during cross-wind gusts. To this end, the supply pressures $p_{a, r}$ and $p_{a, l}$ to the right and left actuators are controlled separately by a pair of Piezo pressure regulators (Hoerbiger, tecno basic PRE-U). The upper and lower actuator slots have the pressure supply $p_{a, u l}$. It is adjusted by a third Piezo pressure regulator. Figure 2 shows a close-up view of the actuator geometry. The pressurized air is accelerated through a duct and exits the actuator through a slot with a width of $w_{\mathrm{S}}=0.3 \mathrm{~mm}$. The actuator jet stays attached to an adjacent rounded surface due to the Coanda effect and is thus redirected towards the base of the bluff body. The momentum coefficient characterizing the actuation amplitude is defined by the following:

$c_{\mu, j}=\frac{A_{a, j} \mathrm{u}_{a, j}^{2}}{A_{B} \mathrm{u}_{\infty}^{2}}$

where $\mathrm{u}_{a, j}$ refers to the jet velocity at the slot exit $j$ with cross-sectional surface $A_{a, j}$. At maximum wind-tunnel velocity, the actuators can achieve an overall momentum coefficient $c_{\mu} \leq 0.063$. A digital signal processor (dSpace, DS $1005 \mathrm{PPC}$ ) running at a sampling frequency of $1 \mathrm{kHz}$ is used for data acquisition, control of the actuators, and real-time simulation of the models for lateral vehicle dynamics and driver behavior.

\subsection{Lateral vehicle dynamics}

The test section is equipped with a novel model support system to replicate lateral vehicle motion in wind-tunnel experiments. The bluff body is mounted on a slender oval pipe that can be rotated and traversed using two magnetic servo linear actuators (Linmot, PS01-37x120) installed below the test section. A telescopic sliding mechanism covers the slot needed for the lateral movement. A six-component force/torque sensor (ATI, FTD Gamma SI-32-2.5, absolute measurement accuracy better than $1 \%$ of full scale span) is installed inside the model and monitors the transient forces and moments acting on the body. Two tri-axial accelerometers (Pololu, MMA7361L) positioned at the vehicle's front and rear axle locations measure the model's angular and lateral acceleration. Based on these measurements, the balance readings are compensated for inertial forces and moments during dynamic motion and serve as input variables for a real-time simulation of lateral vehicle dynamics and driver behavior.

\subsubsection{Single-track model}

The main characteristics of lateral vehicle dynamics can be described by the linear single-track model (Mitschke and Wallentowitz 2004). As shown in Fig. 3, the wheels of the front and rear axles are merged to single, massless wheels at the center of each axle, respectively. The motion of the vehicle with mass $m$ and moment of inertia $J_{z}$ is assumed to be restricted to the horizontal plane, with a center of gravity located at road level at a distance of $L_{f}$ and $L_{r}$ from the front and rear axles. The tire properties and steering stiffness are represented by linearized front and rear cornering stiffness

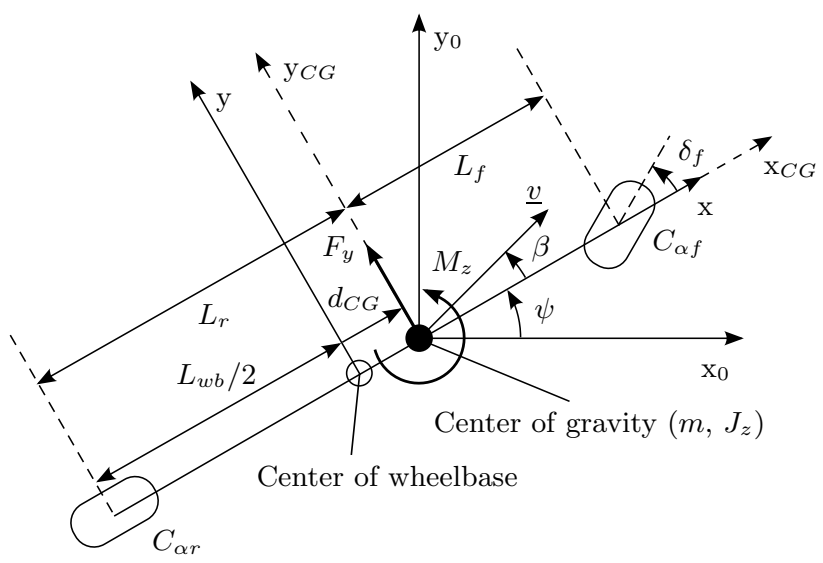

Fig. 3 Single-track model 
coefficients $C_{\alpha f}$ and $C_{\alpha r}$. In the classic single-track model, the front wheel angle $\delta_{\mathrm{f}}$ is the only input variable. Here, we substitute it by the steering angle $\delta=i_{s} \delta_{f}$, with steering gear transmission ratio $i_{s}$. As described by Sackmann and Trächtler (2003), the model is augmented by additional input variables $F_{y}$ and $M_{z}$, accounting for the side force and yaw moment acting on the vehicle's center of gravity due to cross wind. Assuming a constant velocity v and small values of the yaw angle $\psi$, side-slip angle $\beta$, and steering angle $\delta$, linearizing the equations for translational and rotational balance of the vehicle yields

$\dot{\beta}=a_{11} \beta+a_{12} \dot{\psi}+b_{1} \delta+e_{1} F_{y}$,

$\ddot{\psi}=a_{21} \beta+a_{22} \dot{\psi}+b_{2} \delta+e_{2} M_{z}$,

with coefficients

$a_{11}=-\frac{C_{\alpha r}+C_{\alpha f}}{m \mathrm{v}} ; a_{12}=\frac{C_{\alpha r} L_{r}-C_{\alpha f} L_{f}}{m \mathrm{v}^{2}}-1 ;$

$a_{21}=\frac{C_{\alpha r} L_{r}+C_{\alpha f} L_{f}}{J_{z}} ; a_{22}=-\frac{C_{\alpha r} L_{r}^{2}+C_{\alpha f} L_{f}^{2}}{J_{z} \mathrm{~V}} ;$

$b_{1}=\frac{C_{\alpha f}}{i_{\mathrm{s}} m \mathrm{v}} ; b_{2}=\frac{C_{\alpha f} L_{f}}{i_{s} J_{z}} ; e_{1}=\frac{1}{m \mathrm{v}} ; e_{2}=\frac{1}{J_{z}}$.

For small angles, the lateral acceleration $\mathrm{a}_{l}$ can be approximated by

$\mathrm{a}_{l} \approx(\dot{\psi}+\dot{\beta}) \mathrm{v}$.

Transferring Eqs. (4), (5), and (7) into the Laplace domain results in

$\mathrm{a}_{l}(s)=G_{\mathrm{a}_{l} \delta}(s) \delta(s)+\boldsymbol{G}_{\mathrm{a}_{l} d}(s)\left[\begin{array}{c}F_{y}(s) \\ M_{z}(s)\end{array}\right]$,

with the Laplace variable $s$ and the transfer functions $G_{\mathrm{a}_{1} \delta}(s)$ and $\boldsymbol{G}_{\mathrm{a}_{l} d}(s)$ for the vehicle's lateral acceleration response to steering input $\delta$ and to disturbance inputs $\underline{d}$. Note that the disturbances side force $F_{y}$ and yaw moment $M_{z}$ are defined here with respect to the center of gravity as is common in driving dynamics. For the real-time simulation of the lateral vehicle dynamics, they are calculated from the measured aerodynamic side force $S$ and yaw moment $N$ by

$F_{\mathrm{y}}=S$ and $M_{\mathrm{z}}=N-d_{C G} S$,

where $d_{C G}$ denotes the distance of the center of gravity from the center of the wheelbase.

\subsubsection{Driver model}

As pointed out by Wagner (2004) and Krantz (2011), the driver's reaction to side-wind disturbances plays an important role when assessing a vehicle's cross-wind sensitivity. Here, we use a model of a virtual driver proposed by Risse (1991). The driver can thereby be represented by a simple linear controller that adjusts the steering angle $\delta$, as shown in Fig. 4. Based on the perceived lateral displacement $\mathrm{y}_{l}$, velocity $\mathrm{v}_{l}$, and acceleration $\mathrm{a}_{l}$, the driver predicts the future motion of the vehicle over a time horizon $T_{P}$, as depicted in the dashed box in Fig. 4. The transfer function

$M_{R}(s)=V_{M} \frac{1}{1+T_{I} s} e^{-s \tau}$

represents the driver's behavior in the frequency domain, with gain $V_{M}$, time constant $T_{I}$, and delay time $\tau$. These parameters are adapted by the driver to the individual vehicle properties, such that the cross-over frequency $f_{c}$ and the phase reserve $\phi_{r}$ of the open driver-vehicle control loop lie within the range $0.3 \mathrm{~Hz}<f_{c}<0.5 \mathrm{~Hz}$ and $30^{\circ}<\phi_{\mathrm{r}}<40^{\circ}$, respectively. For the detailed calculation, please refer to Risse (1991).

Mitschke and Wallentowitz (2004) expand the controller architecture of the virtual driver by an additional feedforward transfer function for cross-wind disturbance compensation. Here, we reformulate this feedforward part as a transfer function matrix:

$\boldsymbol{M}_{d}(s)=\left[\frac{k_{\delta F_{y}}}{1+T_{S} s} e^{-s \tau} \frac{k_{\delta M_{z}}}{1+T_{S} s} e^{-s \tau}\right]$,

with a time constant $T_{S}=T_{I}$ and the same time delay $\tau$ as in the feedback part of the driver. The gains $k_{\delta F_{y}}$ and $k_{\delta M_{z}}$ relate to the steady-state steering angle $\delta$ needed to compensate for constant disturbances $F_{y}$ and $M_{z}$ acting on the vehicle, respectively. A second-order Padé approximation of the time delay $\tau$ yields the overall control law of the virtual driver:

$$
\begin{aligned}
\delta= & {\left[\frac{-V_{M}}{1+T_{I} s} \frac{-V_{M}}{1+T_{I} s} T_{P} \frac{-V_{M}}{1+T_{I} s} \frac{T_{P}^{2}}{2}\right] G_{\text {delay }}(s)\left[\begin{array}{c}
\mathrm{y}_{l} \\
\mathrm{v}_{l} \\
\mathrm{a}_{l}
\end{array}\right] } \\
& +\left[\frac{k_{\delta F_{y}}}{1+T_{S} s} \frac{k_{\delta M_{z}}}{1+T_{S} s}\right] G_{\text {delay }}(s)\left[\begin{array}{c}
F_{y} \\
M_{z}
\end{array}\right],
\end{aligned}
$$

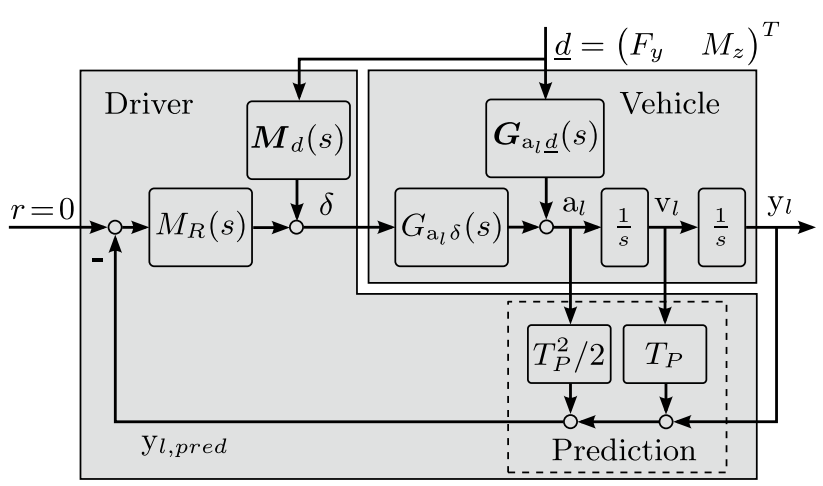

Fig. 4 Driver-vehicle control loop for disturbance compensation. Adapted from Risse (1991) and Mitschke and Wallentowitz (2004) 
with

$G_{\text {delay }}(s)=\frac{1-\frac{\tau}{2} s+\frac{\tau^{2}}{12} s^{2}}{1+\frac{\tau}{2} s+\frac{\tau^{2}}{12} s^{2}}$.

Combining Eqs. (8) and (12) and replacing side force and yaw moment with their nondimensional coefficients $c_{S}$ and $c_{N}$ result in

$\mathrm{a}_{l}(s)=G_{\mathrm{a}_{l} c_{S}}(s) c_{S}(s)+G_{\mathrm{a}_{l} c_{N}}(s) c_{N}(s)$

for the lateral acceleration response of the overall drivervehicle system. As described in Sect. 5, its characteristics are taken into account in the design of the closed-loop controller to improve the lateral dynamic response to cross-wind gusts.

\subsubsection{Implementation and scaling to wind-tunnel conditions}

The values of the coefficients for the single-track model and virtual driver are given in Table 1 . They describe a typical delivery van of the European 3.5t-class with medium load, which is similar in shape and dimensions to the 3D bluff body studied here. To match the smaller model dimensions and the free-stream velocity in the wind tunnel, these coefficients are scaled according to their units, such that nondimensional time $t^{*}=t \mathrm{v} / L$ and nondimensional length $\mathrm{x}^{*}=\mathrm{x} / L$ remain equal for the real van and the model vehicle. Here, the lateral dynamics of the real vehicle at a driving speed $\mathrm{v}_{\text {real }}=120 \mathrm{~km} / \mathrm{h} \approx 33.3 \mathrm{~m} / \mathrm{s}$ is simulated at a wind-tunnel free-stream velocity $\overline{\mathrm{u}}_{\infty}=\mathrm{v}=15.2 \mathrm{~m} / \mathrm{s}$, corresponding to $R e_{L}=4 \times 10^{5}$.

Table 1 Coefficients of single-track model and virtual driver

\begin{tabular}{ll}
\hline Vehicle & Driver \\
\hline$L=5.6 \mathrm{~m}$ & $\tau=0.2 \mathrm{~s}$ \\
$L_{w b}=3.2 \mathrm{~m}$ & $T_{S}=0.2 \mathrm{~s}$ \\
$L_{f}=1.71 \mathrm{~m}$ & $T_{I}=0.7 \mathrm{~s}$ \\
$L_{r}=1.49 \mathrm{~m}$ & $T_{P}=0.7244 \mathrm{~s}$ \\
$d_{C G}=-0.107 \mathrm{~m}$ & $\phi_{r}=35^{\circ}$ \\
$m=3000 \mathrm{~kg}$ & $\omega_{c}=0.35 \cdot 2 \pi \mathrm{rad} / \mathrm{s}$ \\
$J_{z}=7300 \mathrm{~kg} \mathrm{~m}{ }^{2}$ & $V_{M}=0.4017 \% \mathrm{~m}$ \\
$C_{\alpha f}=157500 \mathrm{~N} / \mathrm{m}$ & \\
$C_{\alpha r}=302500 \mathrm{~N} / \mathrm{m}$ & \\
$i_{s}=17.5$ & \\
\hline
\end{tabular}

\section{Flow characteristics}

\subsection{Unsteady cross-wind gust response}

The flow around the bluff body at zero yaw separates at the rear edges and forms a highly turbulent, three-dimensional wake. This leads to a low time-averaged base pressure coefficient $\bar{c}_{p, b} \approx-0.12$ and a high mean drag coefficient $\bar{c}_{D} \approx 0.43$.

The transient responses of force, moment, and pressure coefficients are shown in Fig. 5 for a gust with a maximum cross-wind angle of $\beta_{w} \approx 11^{\circ}$. The depicted time series are phase-averaged over ten identical experiments. The gust begins at $t^{*}=t \mathrm{u}_{\infty} / L \approx 0$ with an increase in normalized total pressure fluctuation $p_{t}^{\prime} / \bar{p}_{t}$. Here, the total pressure measured by the five-hole probe is decomposed by $p_{t}(t)=\bar{p}_{t}+p_{t}^{\prime}(t)$ into a steady mean component $\bar{p}_{t}$ and an unsteady component $p_{t}^{\prime}(t)$. The cross-wind angle starts to increase at $t^{*} \approx 3$, approximately when the total pressure reaches its maximum. The response of the drag coefficient is characterized by a significant delay with respect to cross-wind angle. Furthermore, its transient evolution correlates very well with the base pressure coefficient, indicating that the increase in drag is mostly caused by a modification of the wake during the gust. By contrast, the cross-wind gust response of side-force and yaw-moment coefficients is much faster. Their increase is mostly caused by pressure changes at the front of the bluff body's sides that are immediately affected by the cross-wind angle $\beta_{w}$, see $c_{p_{11}}$ and $c_{p_{17}}$. In comparison, the response of the pressure readings $c_{p_{4}}$ and $c_{p_{24}}$ at the rear sides is much smaller and occurs delayed by approximately 1 convective time unit. This causes a small delay in the build-up of the sideforce coefficient during the gust and an overshoot of the yaw-moment coefficient with respect to the cross-wind angle.

In an application to a real vehicle, the force and moment coefficients would not be available as measurement variables. However, the flow characteristics indicate that the main effects can be estimated from surface-pressure measurements. To this end, surrogate variables $\hat{c}_{D}, \hat{c}_{S}$, and $\hat{c}_{N}$ are calculated from a weighted sum of the pressure coefficients $c_{p, b}, c_{p_{4}}, c_{p_{11}}, c_{p_{17}}$, and $c_{p_{24}}$, located on the bluff body's base and sides, respectively. Their weighting parameters were determined by linear regression from a series of steady-state measurements for several Reynolds numbers, cross-wind angles, and actuation amplitudes (Pfeiffer 2016). As can be seen from Fig. 5, the surrogate variables (plotted in red) match the transient evolution of the force and moment coefficients (plotted in blue) very well. They are thus well suited as measurement variables for the closed-loop controller. 

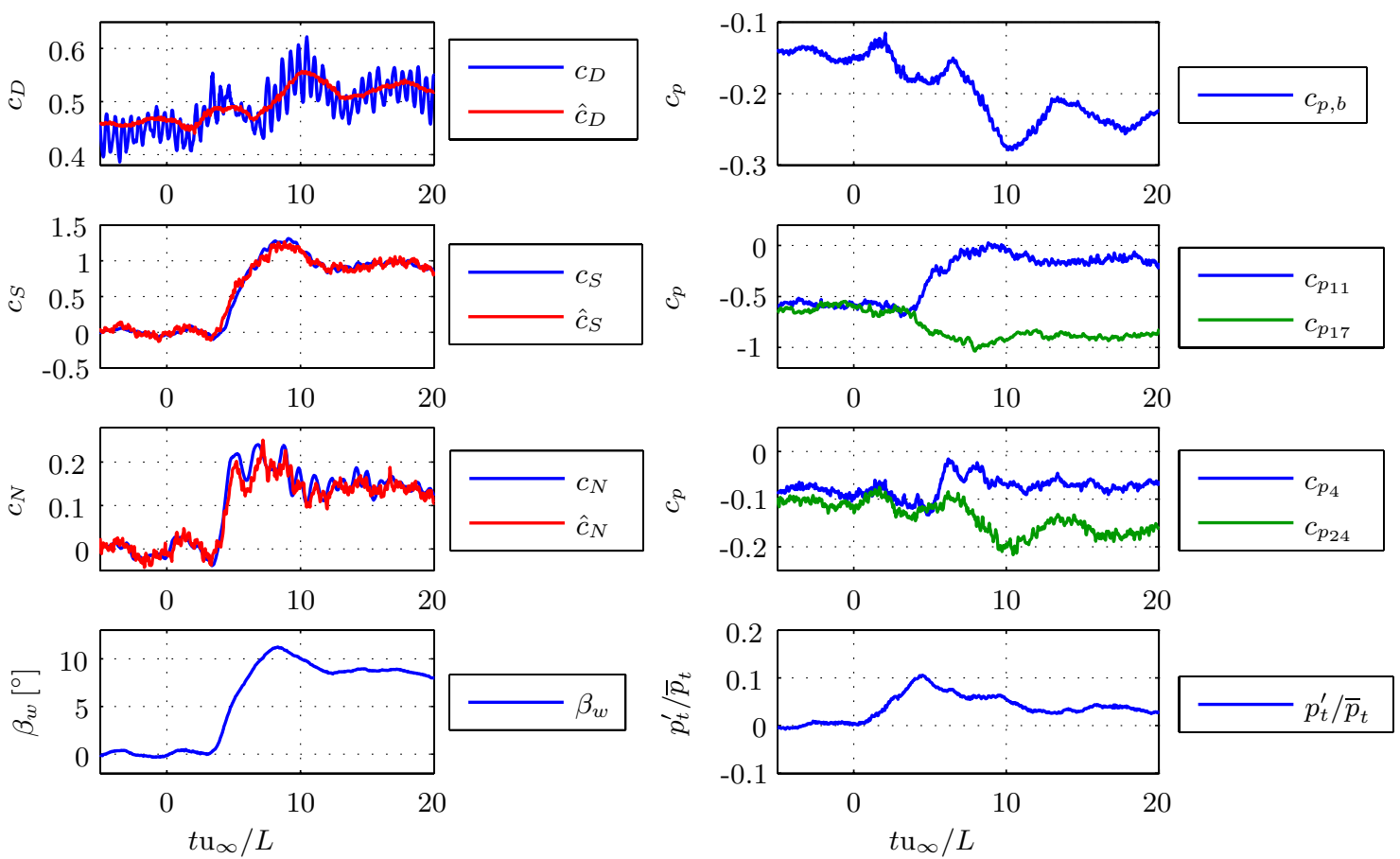

Fig. 5 Phase-averaged time series of transient force and moment coefficients (left) and of selected pressure coefficients (right) for a gust with a maximum cross-wind angle $\beta_{w} \approx 11^{\circ}$

\subsection{Actuated flow}

As already stated in Pfeiffer and King (2012), the most efficient drag reduction at zero yaw is achieved by symmetric actuation with an overall momentum coefficient of $c_{\mu} \approx 0.02$. Due to the Coanda effect, the blown air stays attached to the rounded surfaces at the actuator exit slots and deflects high-momentum free-stream fluid towards the base. This increases the base pressure coefficient and, therefore, reduces drag, as can be seen from the steady-state maps in Fig. 6. However, the actuation becomes less effective with increasing momentum coefficient. Note that the measurement of the drag coefficient also includes the thrust generated by the Coanda jets. Therefore, simply evaluating the achieved drag reduction is not a suitable way to rate the efficiency of an AFC strategy. As suggested by Krentel et al. (2010), we thus calculate the normalized net power savings by taking the power of each actuator jet

$P_{a, j}=\frac{1}{2} \dot{m}_{a, j} \mathrm{u}_{a, j}^{2}=\frac{\rho}{2} A_{a, j} \mathrm{u}_{a, j}^{3}$

into account. Here, $\dot{m}_{a, j}$ and $\mathrm{u}_{a, j}$ denote the mass flow and the jet velocity at each actuator slot $j$, respectively. Overcoming the drag force $D_{0}$ at driving speed $\mathrm{u}_{\infty}$ requires the power

$P_{0}=D_{0} \mathrm{u}_{\infty}=\frac{\rho}{2} c_{D_{0}} A_{B} \mathrm{u}_{\infty}^{3}$.
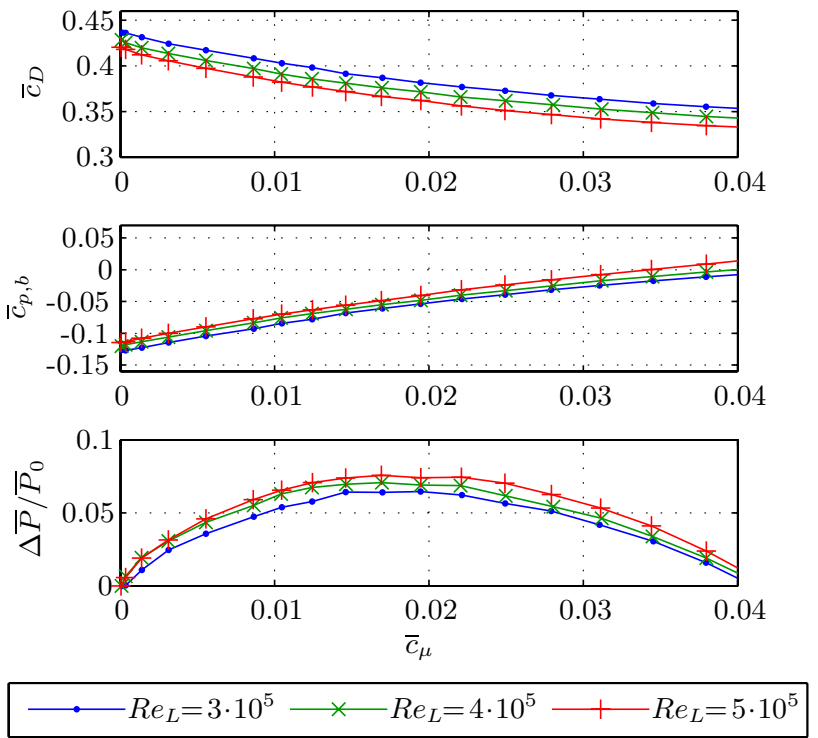

Fig. 6 Steady-state maps of drag coefficient $\bar{c}_{D}$, base pressure coefficient $\bar{c}_{p, b}$, and normalized power savings $\Delta \bar{P} / \bar{P}_{0}$ for symmetric blowing at $\beta_{w}=0^{\circ}$ at several Reynolds numbers 
The normalized net power savings can then be calculated by

$\frac{\Delta P}{P_{0}}=\frac{\Delta c_{D}}{c_{D_{0}}}-\frac{P_{a, r}}{P_{0}}-\frac{P_{a, l}}{P_{0}}-\frac{P_{a, u l}}{P_{0}}$,

where $\Delta c_{D}$ denotes the drag reduction achieved by AFC. $P_{a, r}, P_{a, l}$, and $P_{a, u l}$ represent the power of the right, left, and upper and lower Coanda jets, respectively. The graph at the bottom of Fig. 6 indicates maximum net power savings of approximately $8 \%$ for a momentum coefficient of $c_{\mu} \approx 0.02$ at a drag reduction of $15 \%$.

Cross-wind compensation requires asymmetric actuation with different blowing rates at the windward and leeward sides. The respective maps are shown in Fig. 7 for $\beta_{w}=0^{\circ}$ and $\beta_{w}=10^{\circ}$. Here, we vary the normalized blowing velocities $\mathrm{u}_{a, r}^{*}=\mathrm{u}_{a, r} / \mathrm{u}_{\infty}$ and $\mathrm{u}_{a, l}^{*}=\mathrm{u}_{a, l} / \mathrm{u}_{\infty}$ at the right (windward) and left (leeward) actuators, respectively. Whereas side force and yaw moment depend almost linearly on the actuation amplitude, the drag coefficient shows a highly nonlinear behavior, which additionally depends on cross-wind angle. In addition, note that asymmetric actuation has a contrary effect on side-force and yaw-moment coefficients: Reducing the yaw moment under cross-wind conditions requires a higher blowing rate $\mathrm{u}_{a, l}^{*}$ at the leeward (i.e., left) actuator, but this increases the side force slightly (see Fig. 7e, f, respectively). All these partly opposing dependencies have to be taken into account in the control design to reduce drag efficiently while simultaneously improving the vehicle's cross-wind sensitivity.

\section{Model identification}

The feedback controller for the bluff body is designed based on dynamic black-box models identified from experimental data. This approach has been used successfully in many previous applications of closed-loop AFC (see, e.g., Pastoor et al. 2008; Pfeiffer and King 2012). Here, we identify separate models for the actuator dynamics and the actuated flow dynamics to describe their respective effect on the overall plant dynamics.

\subsection{Actuator dynamics}

The three actuator submodels $i=1 \ldots 3$ receive the desired normalized blowing velocities as input variables $u_{a, i}=\mathrm{u}_{a_{i}, d e s} / \mathrm{u}_{\infty}$, whereas their output variables $y_{a, i}=\mathrm{u}_{a_{i} j e t} / \mathrm{u}_{\infty}$ are defined for the instantaneous nondimensional blowing velocities at the actuator slot exits. Actuators 1 and 2 correspond to the right and left Coanda slots, whereas index 3 designates the upper and lower slots, which are driven as a single actuator. The identification experiments are carried out at several operating points by varying the input variables with a pseudo-random binary signal (PRBS) and recording the output variables. The actuator dynamics do not change significantly over the relevant operating regime, and linear discrete-time single-input single-output (SISO) models provide a good fit of the experimental data. The models are identified separately for each of (a)

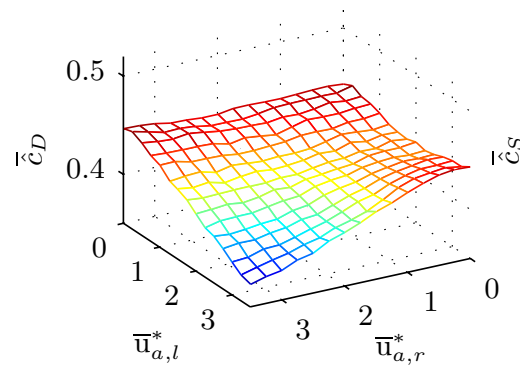

(d)

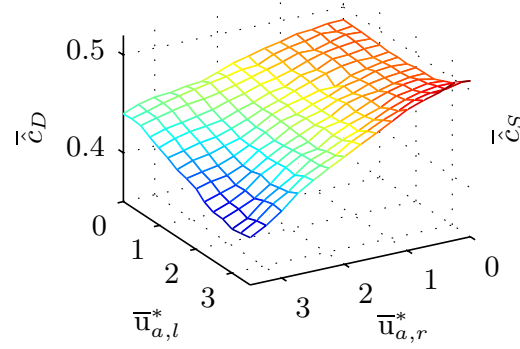

(b)

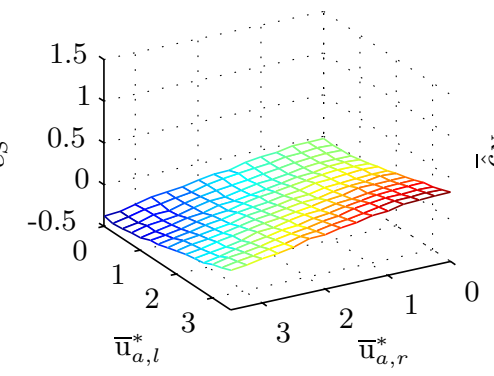

(e)

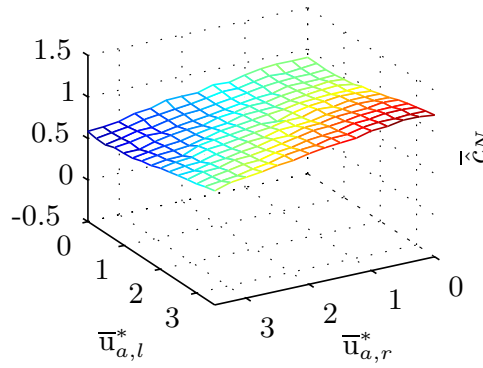

(c)

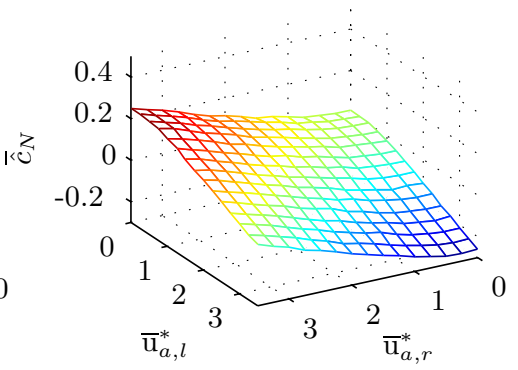

(f)

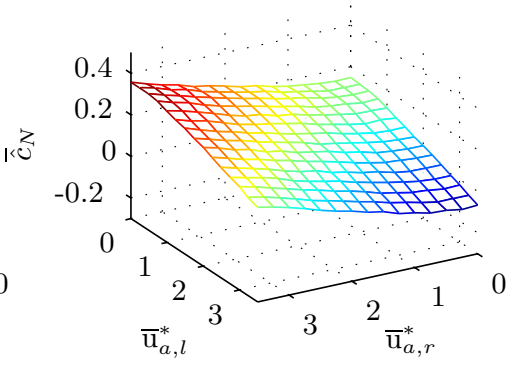

Fig. 7 Steady-state maps of the coefficients for drag $\overline{\hat{c}}_{D}$, side force $\overline{\hat{c}}_{S}$, and yaw moment $\overline{\hat{c}}_{N}$ for asymmetric blowing at a cross-wind angle a-c $\bar{\beta}_{w}=0^{\circ}$ and $\mathbf{d}-\mathbf{f} \bar{\beta}_{w}=10^{\circ}, R e_{L}=4 \times 10^{5}$ 
the three actuators with the prediction error method (PEM) implemented in MATLAB (2007) and have the structure

$\underline{x}_{a, i}(k+1)=\boldsymbol{A}_{a, i} \underline{x}_{a, i}(k)+\underline{b}_{a, i} u_{a, i}\left(k-n_{0}\right)$,

$y_{a, i}(k)=\underline{c}_{a, i}^{T} x_{a, i}(k)$,

with $\underline{x}_{a, i} \in \mathbb{R}^{3}$ and an input delay of $n_{0}=7$ samples at a sampling time $T_{s}=1 \mathrm{~ms}$. All three actuators show first-order low-pass characteristics with a similar bandwidth of about $f_{b w,-3 d B} \approx 20 \mathrm{~Hz}$. The phase response is dominated by the input time delay of $7 \mathrm{~ms}$, which causes important limitations for the achievable closed-loop bandwidth, as discussed in Sect. 5 .

\subsection{Actuated flow dynamics}

The actuated flow model receives the instantaneous actuator jet velocities as input vector

$\underline{u}_{\mathrm{afc}}^{*}=\underline{\mathrm{u}}_{a, j e t}^{*}=\left[\begin{array}{lll}\mathrm{u}_{a_{1}, j e t} / \mathrm{u}_{\infty} & \mathrm{u}_{a_{2}, j e t} / \mathrm{u}_{\infty} & \mathrm{u}_{a_{3}, j e t} / \mathrm{u}_{\infty}\end{array}\right]^{T}$.

The output vector

$y_{\text {afc }}^{*}=\left[\begin{array}{lll}\hat{c}_{\mathrm{D}} & \hat{c}_{\mathrm{S}} & \hat{c}_{\mathrm{N}}\end{array}\right]^{T}$

consists of the surrogate variables for drag, side-force, and yaw-moment coefficients. As the flow characteristics show a strong nonlinear and parameter-dependent behavior, a set of 181 individual models is identified to capture the plant dynamics for a range of actuation amplitudes, Reynolds numbers $3 \times 10^{5} \leq R e_{L} \leq 6 \times 10^{5}$, and cross-wind angles $0^{\circ} \leq \beta_{w} \leq 10^{\circ}$. All models have the same discrete statespace form:

$\underline{x}(k+1)=\boldsymbol{A} \underline{x}(k)+\boldsymbol{B} \underline{u}_{\mathrm{afc}}^{*}(k)$, $\underline{y}^{*} \mathrm{afc}^{*}(k)=\boldsymbol{C} \underline{x}(k)$,

with $\underline{x} \in \mathrm{R}^{4}, \underline{u}_{\mathrm{afc}}^{*} \in \mathbb{R}^{3}$, and $\underline{y}_{\mathrm{afc}}^{*} \in \mathbb{R}^{3}$ at a sampling time $T_{\mathrm{s}}=1 \mathrm{~ms}$. A model order $n_{x}=4$ was found to be sufficient to capture the dynamics of the actuated flow with satisfactory accuracy.

\subsection{Overall plant model for control design}

To obtain an overall plant model of the 3D bluff body for control design, the models for the actuator dynamics and the actuated flow dynamics are connected in series. Furthermore, the output vector is defined as follows:

$\underline{y}=\left[\begin{array}{ll}y_{1} & y_{2}\end{array}\right]^{T}=\left[\begin{array}{lll}\hat{c}_{D} & w_{c_{S}} \hat{c}_{S}+w_{c_{N}} \hat{c}_{N}\end{array}\right]^{T}$.

The second output variable $y_{2}$ is obtained by weighting the estimated side-force and yaw-moment coefficients $\hat{c}_{S}$ and $\hat{c}_{N}$ with constant factors $w_{c_{S}}$ and $w_{c_{N}}$, respectively. These factors are calculated from the relative contribution of the transfer functions $G_{\mathrm{a}_{l} c_{S}}$ and $G_{\mathrm{a}_{l} c_{N}}$ from side-force and yaw-moment coefficients to the overall lateral vehicle acceleration $\mathrm{a}_{l}$. More details on this approach are given in Pfeiffer (2016).

The resulting multiple-input multiple-output (MIMO) statespace models are transferred into matrices of discrete transfer functions $\boldsymbol{G}_{p}(z)$. Setting $z=e^{j \omega T}$ yields the frequency response of a discrete transfer function at a given angular frequency $\omega=2 \pi f$. This is shown in Fig. 8a for the complete set of models $\boldsymbol{G}_{p}$ and the nominal model $\boldsymbol{G}_{n}$ in terms of the maximum and minimum singular values $\sigma_{\max }$ and $\sigma_{\min }$, which correspond to the largest and smallest gain of an MIMO model, respectively. The maximal deviation from the nominal plant can be calculated in terms of the multiplicative output uncertainty:

$l_{o}(\omega)=\max _{\boldsymbol{G}_{p} \in \Pi_{o}} \sigma_{\max }\left(\left(\boldsymbol{G}_{p}-\boldsymbol{G}_{n}\right) \boldsymbol{G}_{n}^{-1}\right)$. (a)

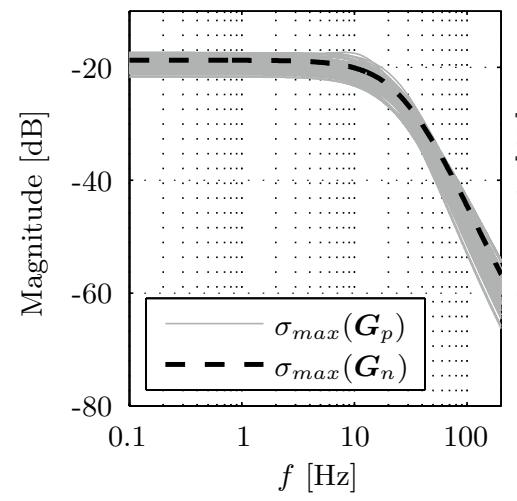

(b)

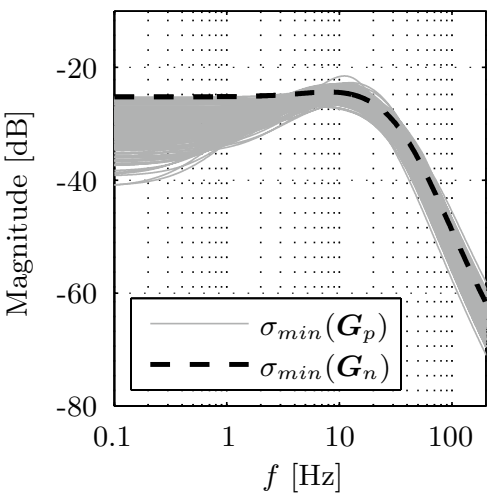

(c)

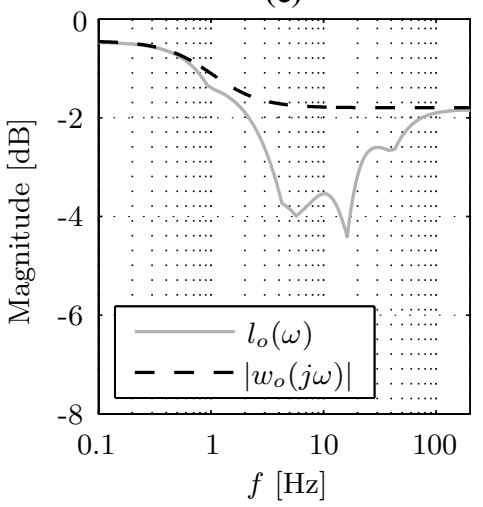

Fig. 8 a Maximal and $\mathbf{b}$ minimal singular values of the set of models $\boldsymbol{G}_{p}$ and of the nominal model $\boldsymbol{G}_{n}$, and $\mathbf{c}$ multiplicative output uncertainty $l_{o}(\omega)$ with the amplitude response of its upper bound $w_{o}(j \omega)$ 
Here, the nominal model $\boldsymbol{G}_{n}(z)$ is derived from one of the black-box models identified at $\operatorname{Re}_{L}=4 \times 10^{5}$. As can be seen from Fig. $8 \mathrm{~b}$, the multiplicative output uncertainty $l_{o}(\omega)$ is below unity for all frequencies $f$. An upper bound for the uncertainty is chosen here as a scalar transfer function

$w_{o}(s)=0.95 \frac{\frac{1}{\omega_{o_{2}}} s+1}{\frac{1}{\omega_{o_{1}}} s+1}$,

with $\omega_{o_{1}}=0.95 \cdot 2 \pi \mathrm{rad} / \mathrm{s}$ and $\omega_{o_{2}}=1.11 \cdot 2 \pi \mathrm{rad} / \mathrm{s}$, such that

$\left|w_{o}(j \omega)\right| \geq l_{o}(\omega), \quad \forall \omega$.

The complete set of models $\Pi_{o}$ is thus described by

$\Pi_{o}: \boldsymbol{G}_{p}=\left(\boldsymbol{I}+\boldsymbol{E}_{o}\right) \boldsymbol{G}, \boldsymbol{E}_{o}=w_{o} \boldsymbol{\Delta}_{o},\left\|\boldsymbol{\Delta}_{o}\right\|_{\infty} \leq 1$,

based on an unstructured multiplicative output uncertainty $\boldsymbol{E}_{o}$ with a normalized uncertainty $\boldsymbol{\Delta}_{o}$. More information concerning this approach is given, for example, by Skogestad and Postlethwaite (2005).

\section{Control design}

The overall controller architecture is shown in Fig. 9. The dynamics of the actuators and of the actuated flow are connected here to an overall plant model as identified in Sect. 4. It thus describes the effect of the desired blowing velocity ratios at the actuators $\underline{u}=\underline{\mathrm{u}}_{a, d e s}^{*}$ on the output variables $y$ that are measured, fed back, and compared against the reference variables $\underline{r}$. Suitable setpoints $\underline{r}$ are calculated by a dynamic reference filter in dependence of the current cross-wind angle $\beta_{w}$. The driver-vehicle model introduced in Sect. 2.3 is simulated in real time based on the measured side-force and yaw-moment coefficients. The computed lateral motion is fed to the linear actuators that move the wind-tunnel model accordingly. Changes in $\beta_{w}$ and $p_{\mathrm{t}}^{\prime} / \bar{p}_{\mathrm{t}}$, as well as the model motion, affect the unsteady aerodynamics of the bluff body. This causes disturbances acting on the output variables $y$, which have to be compensated by the controller.

\subsection{Robust $\boldsymbol{H}_{\infty}$ feedback controller}

The feedback controller has to suppress disturbances due to cross-wind gusts and must track the reference variables $\underline{r}$. Furthermore, it has to guarantee robust stability for the entire set of models. This is achieved with a robust $H_{\infty}$ controller $\boldsymbol{K}$ that is designed based on the plant model identified in Sect. 4. The control synthesis follows standard procedure as described by Skogestad and Postlethwaite (2005) and is summarized here. The output of the controlled plant in the nominal, unsaturated case is given by the following:

$\underline{y}=\boldsymbol{T} \underline{r}+\boldsymbol{S} \underline{d}-\boldsymbol{T} \underline{n}$,

with the complementary sensitivity

$\boldsymbol{T}=\left(\boldsymbol{I}+\boldsymbol{G}_{n} \boldsymbol{K}\right)^{-1} \boldsymbol{G}_{n} \boldsymbol{K}$

representing the closed-loop transfer function with respect to the reference variable $\underline{r}$ and measurement noise $\underline{n}$. The sensitivity

$S=\left(\boldsymbol{I}+\boldsymbol{G}_{n} \boldsymbol{K}\right)^{-1}$

relates to the performance at suppressing disturbances $d$ acting on the output $y$, such as deviations in drag, side-force, and yaw-moment coefficients during cross-wind gusts. Requirements for these closed-loop transfer functions and for the control effort $\boldsymbol{K} \boldsymbol{S}$ are specified via adequate frequency-dependent loopshaping weights. We use a diagonal matrix of transfer functions

$\boldsymbol{W}_{S}=\left[\begin{array}{ll}1 & 0 \\ 0 & 1\end{array}\right] w_{S}(s)$, with $w_{S}(s)=\frac{\frac{1}{M_{S}} s+\omega_{S}}{s+\omega_{S} A_{S}}$,

and $M_{\mathrm{S}}=2, \omega_{\mathrm{S}}=7 \cdot 2 \pi \mathrm{rad} / \mathrm{s}$, and $A_{\mathrm{S}}=1 \times 10^{-4}$ to shape the sensitivity $S$, such that disturbances are suppressed at low frequencies. The weight

$\boldsymbol{W}_{U}=\left[\begin{array}{lll}1 & 0 & 0 \\ 0 & 1 & 0 \\ 0 & 0 & 1\end{array}\right] w_{u}(s)$, with $w_{u}(s)=\frac{0.03\left(s+\omega_{u}\right)}{0.01 s+\omega_{u}}$,

Fig. 9 Controller architecture

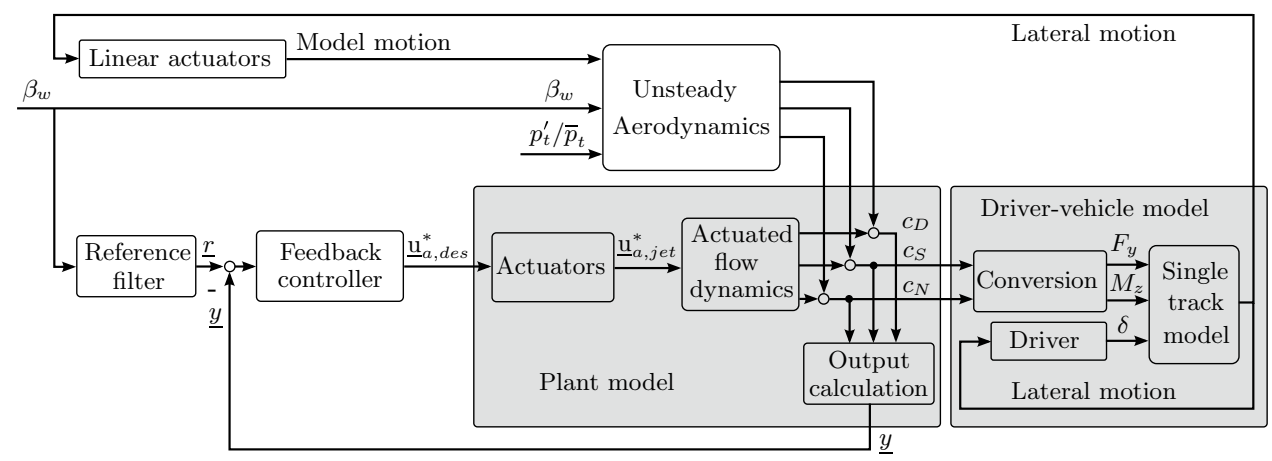


is chosen with $\omega_{u}=50 \cdot 2 \pi \mathrm{rad} / \mathrm{s}$ to limit the control effort $\boldsymbol{K} \boldsymbol{S}$ at high frequencies. Finally, we use a weight

$\boldsymbol{W}_{T}=\left[\begin{array}{ll}1 & 0 \\ 0 & 1\end{array}\right] w_{o}(s)$

for the complementary sensitivity function T. The scalar transfer function $w_{o}(s)$ represents an upper bound for the model uncertainty $l_{o}$. All loopshaping weights are converted to discrete-time transfer functions to facilitate the handling of the time delays at the inputs of the identified model. The controller is obtained by finding

$\min _{K}\left\|\left[\begin{array}{llll}\boldsymbol{W}_{S} \boldsymbol{S} & \boldsymbol{W}_{U} \boldsymbol{K} \boldsymbol{S} & \boldsymbol{W}_{T} \boldsymbol{T}\end{array}\right]^{T}\right\|_{\infty}$.

Figure 10 shows the result of the mixed-sensitivity controller synthesis with the frequency response of the chosen weights. The $H_{\infty}$ controller guarantees robust stability for the entire set of identified models. With respect to disturbance suppression, we obtain a nominal bandwidth of $f_{\mathrm{y}_{1}} \approx 5.6 \mathrm{~Hz}$ for the drag coefficient and $f_{\mathrm{y}_{2}} \approx 6.4 \mathrm{~Hz}$ for the weighted sum of side-force and yaw-moment coefficients. The input time delay of $7 \mathrm{~ms}$ of the actuators causes the so-called waterbed effect. This limits the achievable bandwidth and results in an amplification of disturbances in the range $8 \mathrm{~Hz} \leq f \leq 50 \mathrm{~Hz}$, as can be seen from the frequency response of the sensitivity in the left plot of Fig. 10.

\subsection{Reference filter}

The setpoint $r$ is adjusted online via a dynamic reference filter to ensure an efficient drag reduction under all flow conditions and to minimize the lateral response of the vehicle to the crosswind gust. At first glance, it seems to be an obvious choice to regulate $y_{2}$ to zero to suppress the effect of cross-wind on the lateral vehicle response. This approach was tested in a first version of the setpoint calculation. The results are shown in the steady-state maps in Fig. 11. Here, the reference value $r_{1}$ for the drag coefficient yielding the largest net power savings was determined from a series of steady-state experiments at several constant cross-wind angles $0^{\circ} \leq \beta_{w} \leq 10^{\circ}$. In this case, an efficient drag reduction is, however, only possible for small cross-wind angles $\beta_{w} \leq 4^{\circ}$. Controlling $y_{2}$ to zero at larger angles requires strong asymmetric actuation with a high blowing velocity ratio $\mathrm{u}_{l} / \mathrm{u}_{\infty}$ on the leeward side, thus reducing net power savings.

Instead, a control strategy that suppresses only fast variations of $y_{2}$ is better suited, as the driver can easily adjust for slowly varying cross-wind disturbances. To achieve this, a first-order dynamic reference filter

$r_{2}=F(s) \beta_{w}=\frac{k}{T_{1} s+1} \beta_{w}$, with $T_{1}=\frac{1}{\omega_{F}}$
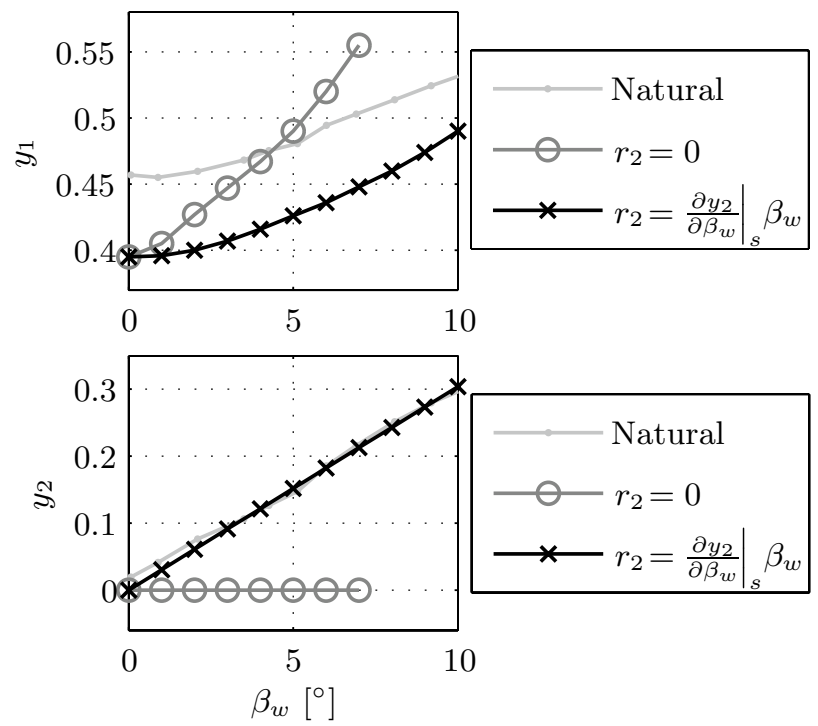

Fig. 11 Reference values for drag coefficient $y_{1}$ and the weighted sum $y_{2}$ of side-force and yaw-moment coefficients relative to the natural flow values for a cross-wind angle variation $0^{\circ} \leq \beta_{w} \leq 10^{\circ}$ (a)

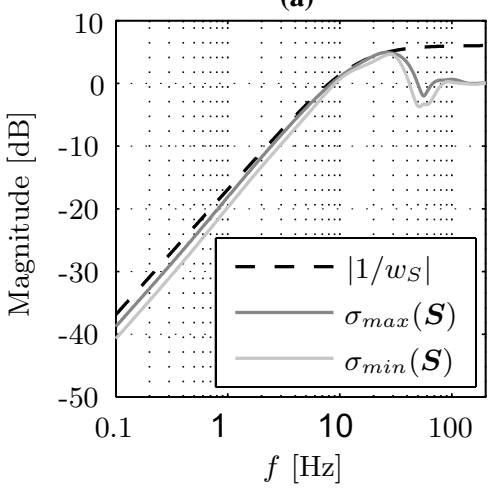

(b)

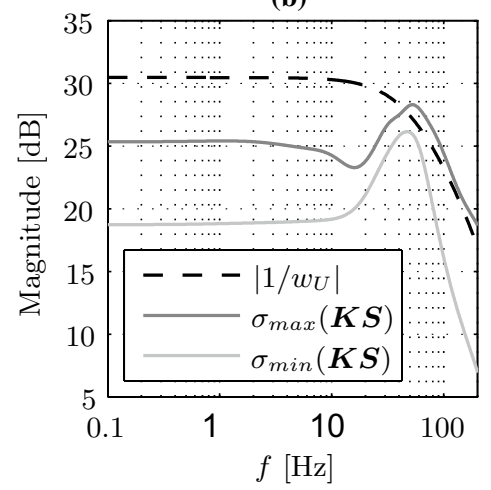

(c)

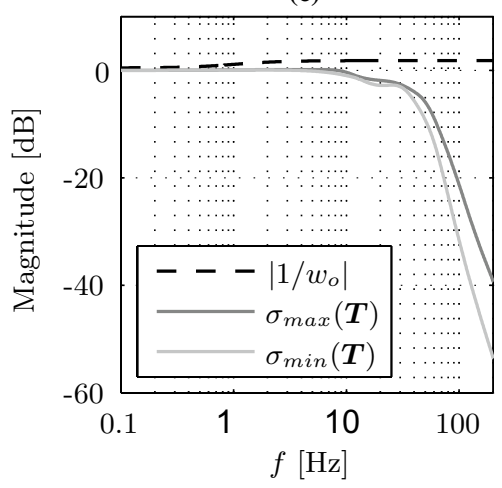

Fig. 10 a Maximum and minimum singular values of the sensitivity $\boldsymbol{S}, \mathbf{b}$ the control effort $\boldsymbol{K S}$, and $\mathbf{c}$ the complementary sensitivity function $\boldsymbol{T}$ together with the magnitude of the inverse of the corresponding scalar weights used for the mixed-sensitivity synthesis of the $H_{\infty}$ controller 
is used here. Its angular roll-off frequency is set to $\omega_{F}=1.2 \cdot 2 \pi \mathrm{rad} / \mathrm{s}$, such that frequencies above $1.2 \mathrm{~Hz}$ are to be suppressed by feedback AFC, whereas slower disturbances are compensated for by the driver. The gain $k$ is chosen equal to the steady-state derivative of $y_{2}$ with respect to $\beta_{w}$ of the natural flow. As can be seen from the lower plot in Fig. 11, this results in a similar steady-state behavior of $y_{2}$ for natural and controlled flow for version 2 of the reference value calculation. Therefore, a more efficient, approximately symmetric actuation can now be applied under constant sidewind conditions, allowing for a lower setpoint for the drag coefficient.

\section{Results}

The developed closed-loop AFC strategy for the 3D bluff body was tested in wind-tunnel experiments with crosswind gusts and real-time replication of the lateral vehicle dynamics and driver behavior. The control objective here is to achieve maximum net power savings by providing an efficient drag reduction while reducing lateral deviation and yaw rate during the gust.

Experimental results are shown in Fig. 12 for the controlled flow relative to the natural flow for gusts with a maximum cross-wind angle $\beta_{w} \approx 10^{\circ}$. As can be seen from Fig. $12 \mathrm{a}$, c, the controller successfully regulates the outputs $y$, so that they follow their setpoint trajectories $r$. This ensures a significant drag reduction relative to the natural, uncontrolled flow. Only at the beginning of the gust is a brief deviation of $y_{1}$ from its setpoint $r_{1}$ visible. The second output variable $y_{2}$ represents the weighted sum of sideforce and yaw-moment coefficients, and approximates their combined effect on the lateral vehicle response. Controlling $y_{2}$ to its reference trajectory $r_{2}$ results in an increase in side force during the cross-wind transient (Fig. 12e). By contrast, the yaw moment, shown in Fig. $12 \mathrm{~g}$, is first (a)

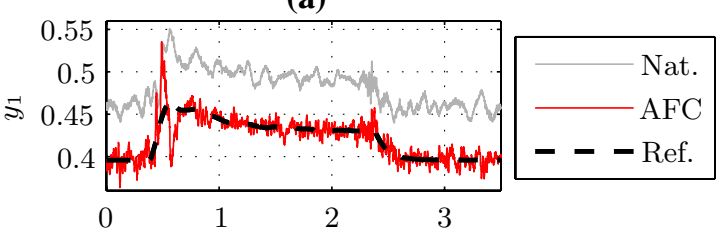

(c)

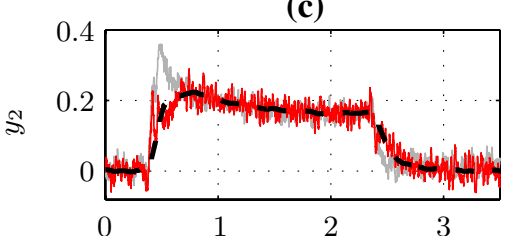

(e)

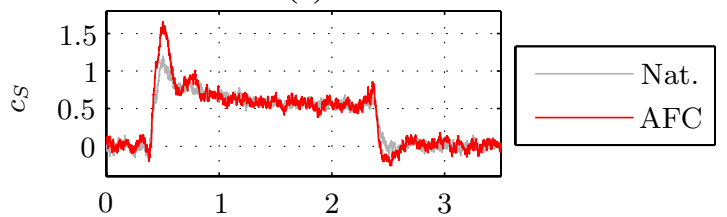

(g)

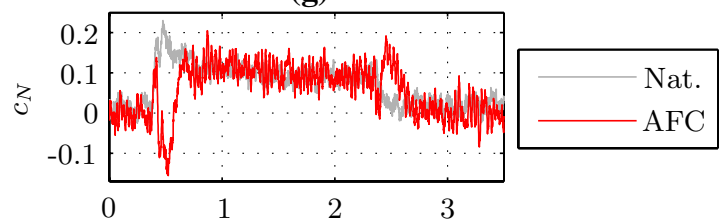

(i)

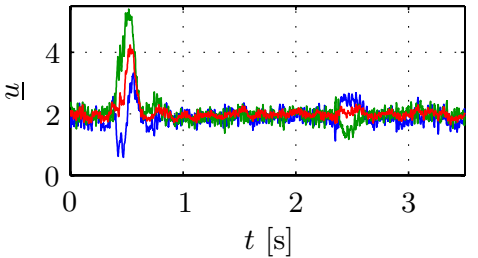

(b)

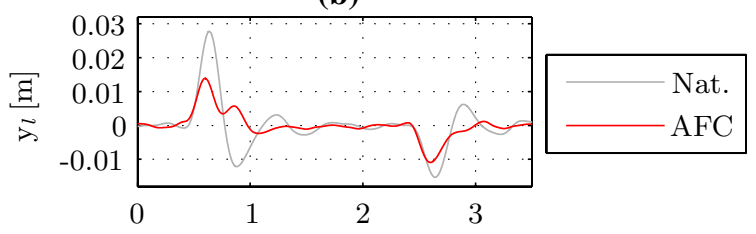

(d)

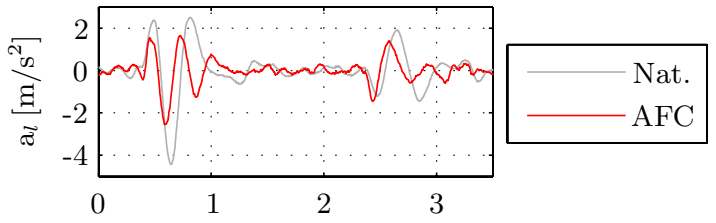

(f)

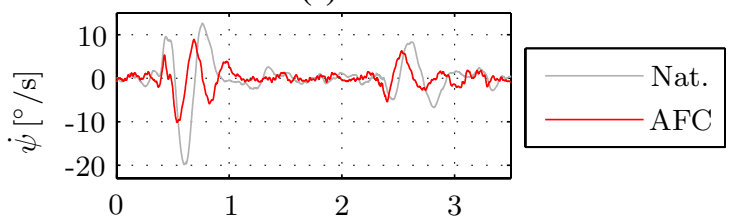

(h)

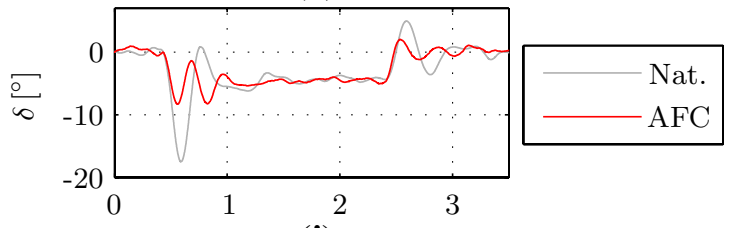

(j)

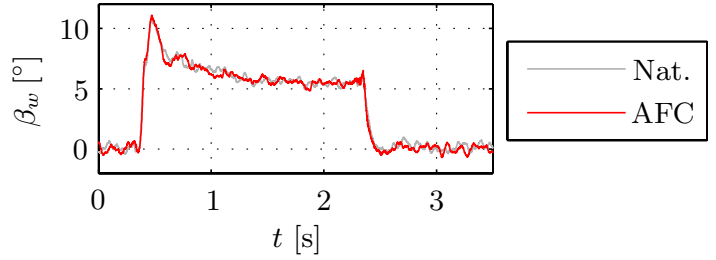

Fig. 12 Results obtained with robust closed-loop AFC relative to the natural cross-wind gust response at $\operatorname{Re}_{L}=4 \times 10^{5}$ 
reduced to negative values to compensate for the effect of the side-force increase on the lateral vehicle response.

When the cross-wind angle $\beta_{w}$ approaches a steady value, both the side-force and yaw-moment coefficients reach values similar to those from the natural flow. Thus, a larger control input is only needed during fast changes of $\beta_{w}$, as in $12 \mathrm{i}$, and the controller suppresses disturbances acting on $y_{2}$ only in a frequency range where the driver is not able to react. A significant reduction of the peaks in lateral deviation $\mathrm{y}_{l}$, lateral acceleration $\mathrm{a}_{l}$, and yaw rate $\psi$ is achieved by closedloop AFC, as can be seen in Fig. 12b, d, f, respectively. This increases driving safety and comfort for the driver, who has to compensate for the effect of steady cross-wind only by slowly turning the steering wheel.

\section{Conclusion}

Our experimental results for a 3D bluff body exposed to cross-wind gusts show how closed-loop active flow control can be used to improve the steady and transient aerodynamic characteristics of road vehicles with respect to drag reduction and side-wind sensitivity. The nonlinear, parameter-dependent characteristics of the actuated flow require a continuous adjustment of the individual blowing velocities of the Coanda actuators located along the rear edges to ensure efficient drag reduction under time-varying flow conditions. This is achieved by applying feedback control, which offers the additional benefit of favorably shaping the frequency response of the aerodynamic force and moment coefficients. Here, we also take the lateral vehicle dynamics and driver behavior into account. This is investigated with a novel model support system that is capable of replicating the lateral vehicle motion in the wind-tunnel based on a realtime simulation of a single-track model and a virtual driver.

The design of the proposed closed-loop AFC strategy is carried out in two steps. First, a multivariable feedback controller for drag, side-force, and yaw-moment coefficients is designed by mixed-sensitivity $H_{\infty}$ loopshaping, such that it provides high tracking performance and fast disturbance suppression. Here, we achieve a nominal bandwidth of 5.6 $\mathrm{Hz}$ for the drag coefficient and $6.4 \mathrm{~Hz}$ for side-force and yaw-moment coefficients. The controller guarantees robust stability for a set of black-box MIMO models identified from experimental data under all relevant flow conditions. In a second step, the control algorithm is augmented by a look-up table for the most efficient setpoint of the drag coefficient and by a dynamic reference filter for side-force and yaw-moment coefficients. This allows the transient aerodynamic characteristics to be adjusted, such that lateral deviation, yaw rate, and steering effort are significantly reduced during cross-wind gusts. Our AFC strategy provides a drag reduction of about $15 \%$, which corresponds to about $8 \%$ net power savings when taking the actuation effort into account. These results demonstrate the advantages of closed-loop AFC for road vehicles over simple open-loop actuation or conventional passive means of aerodynamic optimization that do not offer the possibility of adapting to changing flow conditions.

Acknowledgements This work was funded by the German Research Foundation (DFG) in the context of the research projects Ki 679/9-1 and Ki 679/9-2 "Regelung instationärer Strömungen um stumpfe Körper unter Berücksichtigung der Fahrzeugquerdynamik"

Open Access This article is distributed under the terms of the Creative Commons Attribution 4.0 International License (http://creativeco mmons.org/licenses/by/4.0/), which permits unrestricted use, distribution, and reproduction in any medium, provided you give appropriate credit to the original author(s) and the source, provide a link to the Creative Commons license, and indicate if changes were made.

\section{References}

Aubrun S, McNally J, Alvi F, Kourta A (2011) Separation flow control on a generic ground vehicle using steady microjet arrays. SAE Int J Passenger Cars Mech Syst 51:1177-1187

Brunn A, Wassen E, Sperber D, Nitsche W, Thiele F (2007) Active drag control for a generic car model. In: King R (ed) Active flow control, Notes on Num. Fluid Mech, vol 95. Springer, Berlin, pp 247-259

Dominy R, Ryan A (1999) An improved wind tunnel configuration for the investigation of aerodynamics cross wind gust response. SAE Technical Paper 1999-01-0808

Englar RJ (2001) Advanced aerodynamic devices to improve the performance economics, handling and safety of heavy vehicles. SAE Technical Paper 2001-01-2072

Englar RJ (2004) Pneumatic heavy vehicle aerodynamic drag reduction. In: The aerodynamics of heavy vehicles: trucks, buses, and trains. Lecture notes in applied and computational mechanics, vol 19. Springer, Berlin, pp 277-302

Gilliéron P, Kourta A (2013) Aerodynamic drag control by pulsed jets on simplified car geometry. Exp Fluids 54(1457):1-16

Joseph P, Amandolse X, Aider JL (2012) Drag reduction on the $25^{\circ}$ slant angle ahmed reference body using pulsed jets. Exp Fluids 52:1169-1185

Krajnović S, Basara B (2009) Shape optimization of a double-deck bus for improved aerodynamic stability. In: JUMV international automotive conference with exhibition, Belgrade

Krantz W (2011) An advanced approach for predicting and assessing the driver's response to natural crosswind. $\mathrm{PhD}$ thesis, Universität Stuttgart

Krentel D, Muminović R, Brunn A, Nitsche W, King R (2010) Application of active flow control on generic $3 \mathrm{~d}$ car models. In: King $\mathrm{R}$ (ed) Active flow control II, notes on Num. Fluid Mech, vol 108, pp 223-239

Littlewood RP, Passmore MA (2012) Aerodynamic drag reduction of a simplified squareback vehicle using steady blowing. Exp Fluids 53:519-529

MATLAB (2007) Release 2007b. The MathWorks Inc., Natick

Mitschke M, Wallentowitz H (2004) Dynamik der Kraftfahrzeuge, 4th edn. Springer, Berlin 
Muminović R, Henning L, King R, Brunn A, Nitsche W (2008) Robust and model predictive drag control for a generic car model. In: 4th AIAA flow control conference, AIAA 2008-3859. Reston, VA

Park H, Cho JH, Lee J, Lee DH, Kim KH (2013) Aerodynamic drag reduction of ahmed model using synthetic jet array. SAE Int J Passeng Cars Mech Syst 6(1):1-6

Pastoor M, Henning L, Noack B, King R, Tadmor G (2008) Feedback shear layer control for bluff body drag reduction. J Fluid Mech 608(1):161-196

Pfeiffer J (2016) Closed-loop active flow control for road vehicles under unsteady cross-wind conditions. PhD thesis, TU Berlin

Pfeiffer J, King R (2012) Multivariable closed-loop flow control of drag and yaw moment for a $3 \mathrm{~d}$ bluff body. In: 6th AIAA flow control conference, AIAA 2012-2802. Reston, VA

Pfeiffer J, King R (2014) Linear parameter-varying active flow control for a 3D bluff body exposed to cross-wind gusts. In: 32nd AIAA applied aerodynamics conference, AIAA 2014-2406. Reston, VA
Risse HJ (1991) Das Fahrerverhalten bei normaler Fahrzeugführung. $\mathrm{PhD}$ thesis, TU Braunschweig

Sackmann M, Trächtler A (2003) Nichtlineare Fahrdynamikregelung mit einer aktiven Vorderachslenkung zur Verbesserung des Seitenwindverhaltens. at Automatisierungstechnik 51(12):535-546

Sims-Williams D (2011) Cross winds and transients: reality, simulation and effects. SAE Int J Passenger Cars Mech Syst 4(1):172-182

Skogestad S, Postlethwaite I (2005) Multivariable feedback controlanalysis and design, 2nd edn. Wiley, Chichester

Wagner A (2004) An approach to predict and evaluate the driver's response to crosswind. In: Hucho WH, Wiedemann J (ed) Progress in Vehicle Aerodynamics III - Unsteady Flow Effects, pp 107-120, Expert Verlag, Renningen 